\title{
FRUITONE CPA PARA RETARDAR LA MADURACIÓN EN PIÑA Ananas comosus (L.) Merr., cv. CAYENA LISA, COSECHADA EN PRIMAVERA ${ }^{1}$
}

\author{
ANDRÉS REBOLLEDO-MARTÍNEZ², DANIEL URIZA-ÁVILA², LAUREANO REBOLLEDO-MARTÍNEZ², \\ A. ENRIQUE BECERRIL-ROMÁN ${ }^{3}$, Y LUCERO DEL M. RUIZ-POSADAS ${ }^{4}$
}

\begin{abstract}
RESUMEN -El objetivo fue determinar el efecto de dosis y número de aplicaciones de Fruitone CPA (ácido 2-3 clorofenoxi-propiónico) en el retraso de la maduración, rendimiento y calidad del fruto de piña en el periodo de primavera. El experimento se llevó al cabo en el Campo Experimental Papaloapan, del INIFAP, en Veracruz, México. Se estableció con el cv. Cayena Lisa de México, en un diseño de bloques al azar con cuatro repeticiones; los tratamientos fueron nueve, incluido el testigo sin aplicación, 300, 600, 900 y 1200 mL por hectárea en una aplicación y 600, 900, 1500 y 2100 mL por hectárea divididas en dos aplicaciones iguales, espaciadas ocho días, de Fruitone CPA; los tratamientos se establecieron a los 135 días después de la inducción floral, a 65 días antes de la cosecha del testigo. Los resultados muestran un alto y significativo efecto del Fruitone en el peso de la fruta, aunque no hubo diferencias entre el número de aplicaciones; el contenido de ácido cítrico y los sólidos solubles totales se incrementaron con la aplicación del Fruitone. No se presentaron frutos agrietados ni con Mancha Café interna en ningún tratamiento. El retraso de la cosecha con respecto al testigo varió desde los seis hasta los 21 días, en función de la dosis. Se concluye que el mejor tratamiento fue el de 900 mL de Fruitone CPA por hectárea, en una sola aplicación, el cual incrementó el peso del fruto en un 14\% y retrasó la cosecha 19 días.
\end{abstract}

Palabras clave: Rendimiento, calidad del fruto, producción forzada.

\section{FRUITONE CPA FOR DELAY THE FRUIT MATURITY OF PINEAPPLE Ananas comosus (L) Merr. cv. SMOOTH CAYENNE HARVESTED IN SPRING TIME.}

\begin{abstract}
The objective of this research was to evaluate the effect of rates and applications of Fruitone CPA (acid 2-3 clorofenoxi -propionic) over maturity delay, yield and quality of the pineapple fruit in spring time. The experiment was carried out in the Campo Experimental Papaloapan (INIFAP), in Veracruz State, Mexico. It was established with the cv. Smooth Cayenne, in randomized blocks, design with four replicates; totalizing nine treatments including, the control: 300, 600, 900 and 1200 mL of Fruitone CPA per hectare in one application and 600, 900, 1500 and $2100 \mathrm{~mL}$ per hectare of Fruitone CPA splited into two equal applications separated by eight days. The treatments were applied 135 days after the flower induction and 65 days before the harvest of the control. The results shown a highly significant effect of the Fruitone upon the fruit weight, though there were not differences due to applications; the citric acid content and the total soluble solids in fruits increased with Fruitone. Neither cracked fruits nor marbling disease were presented in any treatment. The maturity was delayed from 6 to 21 days by the control, varying with the dose. It is concluded that the best treatment was $900 \mathrm{~mL}$ of Fruitone CPA per hectare, in one application, which increased 14\% the fruit weight and delayed the harvest in 19 days.
\end{abstract}

Index terms: Yield, fruit quality, production out of season.

\section{INTRODUCCIÓN}

El área piñera de México se caracteriza por tener condiciones contrastantes durante el año: días cortos y frescos en invierno y largos y calurosos en verano; la variación entre el día y la noche es de dos horas y diez minutos. Se localiza entre los 16 y $18^{\circ}$ de latitud norte y a los $95^{\circ} 31^{\prime}$ de longitud oeste; a una altitud de cero a $200 \mathrm{msnm}$; la humedad relativa anual varía entre 50 y $80 \%$ y La temperatura media anual es de $23^{\circ} \mathrm{C}$; el periodo mas frío del año ocurre de diciembre a febrero y el más cálido de abril a junio; la precipitación media anual es de $1200 \mathrm{~mm}$ (Rebolledo et al., 2000).

El Fruitone CPA (ácido 2-3 clorofenoxi-propiónico) es una auxina que permite que la fruta permanezca mas días en la planta (Dalldorf, 1978; Vieira, 1991; Soler 1985) lo cual hace que la cosecha se retrase de una a cuatro semanas en función de la dosis (Soler, 1985; Rebolledo et al., 1993) y el fruto aumente su tamaño y translucidez; y disminuya el contenido de azúcar y la acidez; además la producción de hijuelos se incrementa (IRFA, 1984). Este producto ha sido utilizado también para reducir el tamaño de la corona (Py et al., 1984; Sampaio et al., 1997).

Soler (1990) aplicó Fruitone en el cultivar Cayena Lisa en dosis de $1.5 \mathrm{~L} \mathrm{ha}^{-1}$ al final de la floración y retardó la maduración en siete días, mientras que el peso del fruto se incrementó en $20 \%$. Dosis excesivas pueden traer problemas de agrietamientos de fruto (Soler 1990), lo cual fue constatado por Orona (1992) quien obtuvo el $12 \%$ de fruta agrietada cuando aplicó el producto en abril; mas tarde, Rebolledo y Uriza (1994) encontraron que el porcentaje de frutos agrietados está en función de la dosis y la época del año en que se aplica el producto, al obtener porcentajes de agrietamientos mas altos al increnetar la dosis, en cosechas de noviembre, al inicio del invierno.

1 (Trabalho 045/2001). Recebido: 16/02/2001. Aceito para publicação: 02/05/2002.

2 Investigadores Titulares del Programa de Piña del INIFAP. Apdo Postal 43. C.P. 91641; Cd Isla, Veracruz, México; tel/fax: 287-2 16 82; e-mail: uaa707jc@cirgoc.inifap.conacyt.mx

3 Profesor Investigador Titular de la especialidad de Fruticultura del Colegio de Postgraduados; e-mail: becerril@colpos.colpos.mx

4 Profesora Investigadora Asociada de la especialidad de Botánica del Colegio de Postraduados; lucpo@ colpos.colpos.mx

Rev. Bras. Frutic., Jaboticabal - SP, v. 24, n. 2, p. 354-358, agosto 2002 
Orona (1992), en aplicaciones de Fruitone en abril, encontró un $10 \%$ de frutas con "mancha café". Por otra parte, Rebolledo et al., (1991) evaluaron en el cultivar Cayena Lisa de México diferentes concentraciones de Fruitone y lugar de aplicación; no encontraron diferencias estadísticas significativas entre tratamientos con Fruitone, pero en relación con el testigo, el peso del fruto se incrementó en un $28 \%$ mientras el de la corona se redujo en un $20 \%$. El contenido de azúcar y el contenido de ácido cítrico decrecieron y la relación sólidos solubles totales /acidez aumentó.

En un estudio sobre el comportamiento de Fruitone aplicado en enero, en la misma variedad en una densidad de 30,000 plantas por hectárea, con diferentes niveles de fertilización durante la etapa vegetativa, los frutos tratados presentaron menor contenido de acidez y azúcares así como un incremento en el peso del $28 \%$ y ningún fruto cónico (abotellado), mientras que los frutos no tratados con Fruitone presentaron el $11 \%$ de frutos abotellados. También a medida que se elevaron los niveles de nitrógeno y potasio, el peso se incrementó (Rebolledo et al., 1993).

Se evaluaron dosis de Fruitone en noviembre en una y dos aplicaciones desde 300 hasta $2000 \mathrm{ml} \mathrm{ha}^{-1}$ de Ácido Giberélico (5 y $10 \mathrm{mg} \mathrm{L}^{-1}$ )en invierno en el cultivar Cayena Lisa en 33,000 plantas por hectárea; se encontró que ambos productos tuvieron un efecto retardante sobre la maduración: Fruitone retardó desde 21 hasta 37 días, de acuerdo con la concentración y número de aplicaciones en tanto el Ácido Giberélico solo se retrasó en cinco días. El peso del fruto se incrementó con los dos productos, pero con Fruitone tuvo mejor respuesta. Los contenidos de ácido cítrico se incrementaron en los frutos con Fruitone, lo cual fue contrario a lo encontrado en otros trabajos; asimismo se obtuvo una respuesta desfavorable con las dosis altas de Fruitone al disminuir el contenido de azúcares (Rebolledo y Uriza, 1994). También se evaluó el ácido naftalenacético (ANA) en dosis de 80,100 y $150 \mathrm{mg} \mathrm{L}^{-1}$ y al Fruitone CPA en 50 y $100 \mathrm{mg}$ $\mathrm{L}^{-1}$; no hubo diferencias significativas entre las dosis, pero con respecto al testigo, ANA causó un incremento en peso del fruto del $14 \%$ y el Fruitone del $20 \%$ (Rebolledo et al 1997). Selamat y Rahin (1997) evaluaron concentraciones de sodio 2-3 clorofenoxipropiónico en el cultivar Gandul; la mejor dosis fue la de $1 \mathrm{mg} \mathrm{L}$ ${ }^{1}$; dosis altas del producto redujeron la concentración de sólidos solubles e incrementaron la acidez del fruto; el tamaño de la corona se redujo y la maduración fue retrasada.

El Fruitone CPA actualmente en México está siendo utilizado por muchos productores piñeros en solo una época del año y en muchos casos en dosis inadecuadas ya que la respuesta depende de la época del año. Por tal motivo se estableció un experimento con el objetivo de definir el mejor tratamiento de Fruitone CPA en aplicaciones en el periodo de primavera para retardar la maduración e incrementar el rendimiento del fruto sin deteriorar su calidad.

\section{MATERIALES Y MÉTODOS}

El trabajo se desarrolló en el Campo Experimental Papaloapan, del Instituto Nacional de Investigaciones Forestales, Agrícolas y Pecuarias (INIFAP), ubicado en el municipio de Isla, al sur del estado de Veracruz, centro de la principal región productora de piña en México. Se localiza a $18^{\circ} 06^{\prime}$ de Latitud Norte y a los $95^{\circ} 31^{\prime}$ de Longitud Oeste, a $50 \mathrm{msnm}$. El clima es un $\mathrm{Aw}_{\mathrm{o}}$, clasificado como cálido subhúmedo (García, 1988). La temperatura media es de $23^{\circ} \mathrm{C}$; el período mas frío del año ocurre de diciembre a febrero con promedios de temperaturas mínimas y máximas en esos meses de 16.2 y $28.9^{\circ} \mathrm{C}$ respectivamente, con mínima absoluta de $6^{\circ} \mathrm{C}$; la época más caliente del año se presenta en abril, mayo y junio, con temperaturas máximas y mínimas para ese periodo de 36.7 y $20.9^{\circ} \mathrm{C}$ respectivamente, con máxima absoluta de $44^{\circ} \mathrm{C}$. La precipitación anual es de $1200 \mathrm{~mm}$, de los cuales el $7 \%$ caen de enero a mayo, el $80 \%$ de junio a octubre y el $13 \%$ en noviembre y diciembre. Los suelos predominantes son Acrisoles y Cambisoles, con textura migajón arenosa, pobres en materia orgánica y nutrimentos y con un $\mathrm{pH}$ de 4.5 , sin problemas de salinidad.

Para el experimento se utilizó material vegetativo tipo "clavo" de $600 \mathrm{~g}$ del cultivar Cayena Lisa de México, plantado en febrero, con una densidad de 35,000 plantas por hectárea a doble hilera. El diseño experimental utilizado fue el de bloques completos al azar con cuatro repeticiones; la parcela experimental constó de dos hileras dobles de piña de $10 \mathrm{~m}$ con un total de 40 plantas consideradas como parcela útil. La diferenciación floral ocurrió de manera natural en diciembre de 1995 y la primera aplicación de los tratamientos se hizo a mediados de abril de 1996 a los 135 días después de la iniciación floral y 65 días antes de la cosecha del testigo; mientras que la segunda aplicación se efectuó ocho días después de la primera. La cosecha del testigo se realizó el 20 de junio. Se evaluaron un total de ocho tratamientos además del testigo sin aplicación: cuatro dosis de Fruitone CPA (8.0 \%i.a.) comercial por hectárea en una sola aplicación: a) $300 \mathrm{ml}$; b) 600 $\mathrm{ml}$; ) $900 \mathrm{ml}$ d) $1200 \mathrm{ml}$; y cuatro dosis por hectárea del mismo producto dividida en dos aplicaciones iguales: a) $600 \mathrm{ml}$; c) 900 $\mathrm{ml}$; ) $1500 \mathrm{ml} \mathrm{y}$;) $2100 \mathrm{ml}$. Se aplicaron $40 \mathrm{ml}$ de la solución dirigida directamente a la fruta, sin bañar la corona; la hora de aplicación fue de 9:00 a 11:00 a.m.

Las variables medidas fueron: Peso de fruto; porcentaje de frutos agrietados; porcentaje de frutos manchados internamente; dimensiones del fruto y de corona; sólidos solubles totales (grados brix con un refractómetro); contenido de acidez (por titulación con $\mathrm{NaOH}, \mathrm{AOAC}, 1990$ ); translucidez (Soler,1992) y; días de retraso de la cosecha.

\section{RESULTADOS Y DISCUSIÓN}

En el cuadro 1 se aprecia el alto y significativo efecto del Fruitone en el peso de la fruta; con excepción del testigo y las dosis de $300 \mathrm{ml}$ en una aplicación y $600 \mathrm{ml}$ en dos aplicaciones, el resto de los tratamientos estudiados tuvieron un comportamiento estadístico similar por lo que con solo realizar una aplicación $600 \mathrm{ml}$ por hectárea o mas, es suficiente para lograr un incremento en el rendimiento del ya que dosis mayores aún con dos aplicaciones implican mayor costo; el peso de la corona disminuyó hasta en un $10 \%$ cuando la cantidad del producto aplicado fue mayor, sin haber diferencia estadística entre el número de aplicaciones; menos y tamaño de corona significa mayor aceptación de la fruta (Py et al., 1984; Sampaio et al., 1997) al haber mayor proporción de fruta comestible. Estos resultados no coinciden con lo encontrado en invierno en la 
misma localidad, en donde se logra la misma ganancia de peso del fruto, además de una disminución del peso de la corona, con solamente $500 \mathrm{ml}$ de Fruitone por hectárea en una aplicación (Rebolledo y Uriza, 1994).

El retraso de la cosecha dependió de la dosis utilizada y del número de aplicaciones; en la dosis mas baja con una sola aplicación el efecto para esta variable fue de solo seis días, mismos que obtuvo Soler (1990) con $1.5 \mathrm{~L} \mathrm{ha}^{-1}$, mientras que los demás tratamientos tuvieron retraso desde los 15 hasta los 21 días, situación que varió en función de la dosis y no del número de aplicaciones; por ello también se considera que una aplicación de $900 \mathrm{ml}$ por hectárea es suficiente para lograr el efecto deseado, (Cuadro 1). El retraso es considerado positivo, ya que es otra forma de programar la cosecha para intentar conseguir mejor precio en el mercado nacional o planear los embarques si es para exportación en fresco; Rebolledo y Uriza (1994) encontraron un retraso de 31 días con $900 \mathrm{ml}$ por hectárea de Fruitone, aplicado en noviembre.

En relación con las dimensiones del fruto, Fruitona no tuvo efectos en su longitud, aunque existe la tendencia de aumentar al incrementarse la dosis; el diámetro superior muestra una respuesta significativa a las dosis mas altas, independientemente del número de aplicaciones; esta situación es similar para el diámetro central, en donde solo el testigo y la dosis de 300 por hectárea ml en una aplicación fueron inferiores. El diámetro inferior del fruto manifiesta los mismos resultados que la variable anterior, solo que, aquí también se incluye la dosis de $600 \mathrm{ml}$ en dos aplicaciones (Cuadro 2). Estos resultados se relacionan con los obtenidos para peso del fruto, en donde el testigo presenta los valores mas bajos.

En cuanto a las características de calidad (Cuadro 3), únicamente el testigo presentó el menor contenido de azúcares, lo cual pudiera ser atribuible a las diferentes fechas de cosecha, ya que los frutos con tratamiento permanecieron mas tiempo en la planta y en plena época en que, por las características de temperatura y humedad, la fruta alcanza los más altos niveles de azúcar, contrario a lo que pasa cuando se cosecha en invierno; de todas maneras, el Fruitone no disminuyó los grados brix, dife- rente a lo encontrado en aplicaciones de noviembre y diciembre en donde el producto causa una disminución de los grados brix (Rebolledo et al., 1993; Rebolledo y Uriza, 1994)

El contenido de ácido cítrico en los frutos tratados con Fruitone presentó un ligero incremento de hasta el $10 \%$ en relación con el testigo y con la dosis mas baja, (Cuadro 3), lo cual coincide con los resultados obtenidos por Rebolledo y Uriza (1994) en aplicaciones de noviembre y por Selamat y Rahin (1997) en donde el contenido de ácido cítrico se incrementa con el uso de este regulador; sin embargo los resultados aquí mostrados son contrarios a lo indicado en trabajos establecidos en otras fechas del año, en donde se ha observado que el uso de este regulador reduce drásticamente la acidez del fruto (IRFA, 1984; Rebolledo et al., 1991; Rebolledo et al., 1993). Esta situación podría estar dada por la época del año en que se aplica, debido a las diferencias implícitas en temperatura, fotoperiodo, humedad, nutrición de la planta y sobre todo la etapa de desarrollo en que se encuentra el fruto al momento de la aplicación. La relación grados brix/ acidez no se alteró con la aplicación de Fruitone, y los valores obtenidos están dentro de los parámetros de calidad que se tienen a nivel internacional para esta fruta, los cuales varían entre 20 y 30 con óptimos entre 20 y 25.

No se presentaron problemas por agrietamiento del fruto en esta época de aplicación en ningún tratamiento, incluido el testigo, contrario a lo que ocurre en el invierno, en donde, aún con dosis bajas, se tienen daños considerables (Rebolledo y Uriza 1994) los cuales están en función de la cantidad del producto aplicado (Soler, 1990). Se esperaba encontrar daños por la enfermedad denominada mancha café interna del fruto, sobre todo en dosis elevadas de Fruitone, sin embargo, en este ciclo no hubo condiciones de precipitación, humedad relativa y temperatura favorables para el desarrollo del inóculo y no se obtuvo respuesta con relación a la aplicación del Fruitone, por lo que habría que seguir estudiando específicamente esta variable. Tampoco se tuvieron diferencias entre tratamientos en la translucidez interna de la fruta, por lo que la apariencia de la pulpa fue igual tanto en las diferentes dosis evaluadas como en el testigo.

CUADRO 1 - Peso de fruto y corona y retraso de la maduración de piña con diferentes dosis y número de aplicaciones de Fruitone CPA.

\begin{tabular}{|c|c|c|c|c|}
\hline Tratam ientos & & Peso fruto & Peso corona & Retraso cosecha \\
\hline $\mathrm{N}^{\circ} \mathrm{A} \mathrm{plicaciones}$ & $\mathrm{m} \mathrm{l} \mathrm{por} \mathrm{ha}$ & $(\mathrm{kg})$ & $(\mathrm{g})$ & (día s) \\
\hline $\mathrm{U} n \mathrm{a}$ & 300 & $2.34 \quad b \quad c c c$ & 356 a b & 6 \\
\hline $\mathrm{Una}$ & 600 & $2.51 \mathrm{a} \mathrm{b}$ & 357 a b & 15 \\
\hline $\mathrm{U} n \mathrm{a}$ & 900 & $2.55 \quad a$ & 380 a & 19 \\
\hline $\mathrm{U} n \mathrm{a}$ & 1200 & $2.58 \quad \mathrm{a}$ & $337 \quad b$ & 20 \\
\hline $\mathrm{D}$ o s & 600 & 2.25 & 381 a & 15 \\
\hline $\mathrm{D}$ o s & 900 & 2.45 a b $\quad c$ & 386 a & 18 \\
\hline $\mathrm{D}$ o s & 1500 & $2.50 \quad \mathrm{a} \mathrm{b}$ & 356 a b & 19 \\
\hline $\mathrm{D}$ o s & 2100 & 2.60 & 334 & 21 \\
\hline Testigo & 0 & 2.24 & 373 a & 0 \\
\hline $\mathrm{C} \mathrm{V}(\%)$ & & 5.8 & 6.0 & \\
\hline Sig. Estadística* & & $* *$ & $* *$ & \\
\hline
\end{tabular}

*: Significancia de tratamientos en análisis de varianza $(* *: 1 \%)$

Los tratamientos con la misma letra no difieren estadísticamente (tukey; $\mathrm{p} \leq 0.05$ )

Rev. Bras. Frutic., Jaboticabal - SP, v. 24, n. 2, p. 354-358, agosto 2002 
CUADRO 2 - Dimensiones del fruto de piña en diferentes dosis y número de aplicaciones de Fruitone CPA.

\begin{tabular}{|c|c|c|c|c|c|}
\hline \multicolumn{2}{|c|}{ Tratamientos } & \multirow{2}{*}{$\begin{array}{c}\text { Longitud fruto } \\
(\mathrm{cm})\end{array}$} & \multicolumn{3}{|c|}{ Diámetro fruto $(\mathrm{cm})$} \\
\hline $\mathrm{N}^{0}$ Aplicaciones & $\mathrm{ml}$ por ha & & Superior & Central & Inferior \\
\hline Una & 300 & 17.6 & $10.1 \mathrm{c}$ & $12.5 \mathrm{~b}$ & $11.0 \quad \mathrm{de}$ \\
\hline Una & 600 & 18.2 & $10.5 \mathrm{bc}$ & $12.9 \mathrm{ab}$ & $11.5 \mathrm{bcde}$ \\
\hline Una & 900 & 18.5 & $10.9 \mathrm{ab}$ & $13.2 \mathrm{ab}$ & $12.0 \mathrm{abc}$ \\
\hline Una & 1200 & 18.9 & $10.8 \mathrm{bc}$ & $13.3 \mathrm{ab}$ & $11.7 \mathrm{abcde}$ \\
\hline Dos & 600 & 18.3 & $10.6 \mathrm{bc}$ & $12.9 \mathrm{ab}$ & $11.5 \mathrm{bcde}$ \\
\hline Dos & 900 & 18.1 & $10.7 \mathrm{bc}$ & $13.2 \mathrm{ab}$ & $11.8 \mathrm{abcd}$ \\
\hline Dos & 1500 & 18.7 & $11.0 \mathrm{ab}$ & $13.2 \mathrm{ab}$ & $12.1 \mathrm{ab}$ \\
\hline Dos & 2100 & 18.8 & $11.6 \mathrm{a}$ & $13.7 \mathrm{a}$ & $12.5 \mathrm{a}$ \\
\hline Testigo & 0 & 17.6 & $10.5 \mathrm{bc}$ & $12.5 \mathrm{~b}$ & 10.8 \\
\hline $\mathrm{CV}(\%)$ & & 3.13 & 2.67 & 3.17 & 3.36 \\
\hline Sig. Estadística* & & NS & $* *$ & $* *$ & $* *$ \\
\hline
\end{tabular}

*: Significancia de tratamientos en análisis de varianza (**: 1\%; NS: Diferencia no significativa)

Los tratamientos con la misma letra no difieren estadísticamente (Tukey; $\mathrm{p} \leq 0.05$ )

CUADRO 3 - Características de calidad de la fruta de piña en diferentes dosis y número de aplicaciones de Fruitone CPA.

\begin{tabular}{|c|c|c|c|c|}
\hline \multicolumn{2}{|c|}{ Tratamientos } & \multirow{2}{*}{$\begin{array}{c}\text { SST } \\
\text { Grados brix }\end{array}$} & \multirow{2}{*}{$\begin{array}{l}\text { Ácido cítrico } \\
(\%)\end{array}$} & \multirow{2}{*}{$\begin{array}{c}\text { Relación } \\
\text { SST/acidez }\end{array}$} \\
\hline $\mathrm{N}^{\circ}$ aplicaciones & $\mathrm{ml}$ por ha & & & \\
\hline Una & 300 & $12.8 \mathrm{a}$ & 0.48 & 26.6 \\
\hline Una & 600 & $12.7 \mathrm{ab}$ & 0.53 & 23.9 \\
\hline Una & 900 & $12.5 \mathrm{ab}$ & 0.51 & 24.5 \\
\hline Una & 1200 & $12.6 \mathrm{ab}$ & 0.53 & 23.8 \\
\hline Dos & 600 & $12.3 \mathrm{ab}$ & 0.51 & 24.1 \\
\hline Dos & 900 & $12.3 \mathrm{ab}$ & 0.51 & 24.1 \\
\hline Dos & 1500 & $12.5 \mathrm{ab}$ & 0.52 & 24.0 \\
\hline Dos & 2100 & $12.1 \mathrm{ab}$ & 0.50 & 24.2 \\
\hline Testigo & 0 & $12.0 \mathrm{~b}$ & 0.48 & 25 \\
\hline $\mathrm{CV}(\%)$ & & 2.27 & 9.47 & \\
\hline Sign. Estadística* & & $* *$ & NS & \\
\hline
\end{tabular}

*: Significancia de tratamientos en análisis de varianza (**: 1\%; NS: Diferencia no significativa.

Los tratamientos con la misma letra no difieren estadísticamente (Tukey; $\mathrm{p} \leq 0.05$ ).

SST: Sólidos solubles totales.

\section{CONCLUSIONES}

1 - La respuesta del Fruitone CPA está en función de la dosis utilizada, pero no depende del número de aplicaciones.

2 - Se sugiere como mejor tratamiento el de $900 \mathrm{ml}$ por hectárea en una sola aplicación, considerando el tamaño del fruto y la corona, mayor retraso de la maduración, características de calidad y el costo del producto y su aplicación.

3 - En ningún tratamiento se presentaron problemas por agrietamientos del fruto ni de mancha café, contrario a lo ocurrido en invierno.

\section{REFERENCIAS BIBLIOGRÁFICAS}

AOAC (Association of Official Agricultural Chemist). Official Analytical Chemist. 13. ed. Washington, D.C. 1990. 1023 p.

DALLDORF, D.B. The effect of clorophenoxi-propionamide (Fruitone CPA) on the Smooth Cayenne Pineapple. Citrus and 
Subtropical Fruit Journal, Parklands, n. 534, p. 17-18, 1978.

GARCÍA, E. Modificaciones al sistema de clasificación climática de Koppen. México, 1988. 219 p.

IRFA (Institut de Recherches sur les Fruits et Agrumes). Les reserches sur 1'ananas. Fruits, Paris, v. 42, n. 11: p. 651-654, 1984.

ORONA, M.D. Informe anual de investigaciones realizadas en piña en el Campo Experimental Loma Bonita, México: SARHINIFAP, $1992.25 \mathrm{p}$.

PY, C.; LACOEUILHE, J.J.; TEISSON, C.L'ananas, sa culture, ses produits, techniques agricoles et productions tropicales. Paris: Ed Maisonneuve, 1984. 962

REBOLLEDO M. A.; URIZA, A.D.; REYES, M.J.J. Evaluación de diferentes concentraciones y formas de aplicación de Fruitone CPA en piña. In: CONGRESO NACIONAL CIENCIAS HORTÍCOLAS, 4., 1991. Saltillo.

REBOLLEDO, M. A.; URIZA, A. D.; REBOLLEDO, M. L.. Comportamiento del Fruitone CPA bajo cuatro niveles de fertilización en piña Cayena Lisa. In: CONGRESO LATINOAMERICANO DE PIÑICULTURA, 1., 1993, Cali, Colombia. p. 96-105.

REBOLLEDO, M. A.; URIZA, A. D. Dosis y oportunidades de aplicación de Fruitone y Giberelinas para retrasar la maduración en piña en invierno. In: REUNIÓN DE LA «INTERAMERICAN SOCIETY FOR TROPICAL HORTICULTURE. 10., 1994, Campeche, México. p. 51.

REBOLLEDO, M. A.; URIZA, A.D.; REBOLLEDO, M.L. Dife- rentes concentraciones de ácido giberélico y dos tipos de auxinas para la maduración en piña. In: CONGRESO NACIONAL CIENCIAS HORTÍCOLAS. 7. 1997, Culiacán, México.

REBOLLEDO, M. A.; URIZA A.D.; REBOLLEDO. M.L. The Pineapple in Mexico: Current Status and Prospects. Acta Horticulturae, Wageningen, v. 529, p. 85-88, 2000.

SELAMAT, M. M. RAHIN. A.M. The effect of Fruitone application on the Yield and Fruit Quality of Pineapple cv. Gandul on Deep Peat Soil in Malaysia. Pineapple news, v. 3, n. 1, p. 11-12, 1997.

SAMPAIO, A. C.; CUNHA, R.J.P.; CUNHA. A.R. Influência do nitrogênio, época de plantio e ácido 2, 3 - clorofenoxi propionic sobre a produtividade e época de producao do abacaxizeiro cv. Smooth Cayenne. Revista Brasileira de Fruticultura, Cruz das Almas, v. 19, n.1, p. 169-179, 1997.

SOLER, A. Utilisation du Fruitone CPA comme régulateur de croissance sur l'ananas (Cayenne lisse) en Côte Ivoire.Fruits, Paris, v. 40, n. 1, p. 31-38, 1985.

SOLER, A. Advantages and limits to the use of 3 CPA (2-3 clorophenoxi-propionic acid) in pineapple in Ivory Coast. Fruits, Paris, v. 45, n. 4, p. 357-365, 1990.

SOLER, A. La Piña, Criterios de Calidad. París: CIRAD-IRFA, 1992.p. 17.

VIEIRA, A. Efeito da época de aplicação do ácido clorofenoxipropionico sobre o atraso de maturacao dos frutos de abacaxi da cultivar Pérola. Revista Brasileira de Fruticultura, Cruz das Almas, v. 13, n. 3, p. 259-262, 1994. 